over vertebrate origins is similarly open to dispute. Until recently, teeth were all that betrayed the existence of these animals. Although common enough to be used as biostratigraphic marker fossils, the absence of any other tissue made any systematic treatment impossible.

The discovery in Scotland of the fossil of a long, worm-like animal with recognizably conodont teeth at one end has sparked yet more dispute: they could be chordates related to the hagfish Myxine (R. J. Aldridge, University of Leicester, and D. Briggs, University of Bristol), and there is even some evidence for the presence of chordate-like segmental muscle blocks. But other palaeontologists interpret these structures differently, and argue that the conodonts have affinities with aplacophoran molluscs or nemertean worms, or that they are so different from other forms that they constitute a distinct phylum.

\section{Genetic patterns}

Genetics might provide one way to dispel palaeontological myths once and for all. The realization that distinctive homoeobox genes in animals from different phyla share extensive sequential and structural homology (N. H. Patel et al. Cell 58, 955-968; 1989) was one of the main talking points at the meeting. Palaeontologists have often been wary of claims that phylogenetic problems can better be unravelled by analysis of molecular sequence data than through the fossil record. These data come from structural genes and proteins, and their interpretation is plagued by the same problems that beset anatomical evidence. A protein, after all, is just as much a part of an animal's anatomy as is its skeleton.

But homoeobox genes are concerned with the elaboration of anatomical structures rather than simply the differences between them. The unique body plans that are used to characterize phyla are thought to be rooted in different ontogenetic programmes, but how these differences are established at the molecular level is unclear. One idea is that patterns of duplication in homoeobox genes among various deuterostome taxa (P. Holland, University of Oxford) may add useful evidence with which to reconstruct vertebrate phylogeny, and to judge between competing hypotheses based on purely anatomical evidence.

Ogden Nash's dog had a tail on one end, in common with ichthyosaurs, tunicate tadpole larvae and (arguably) calcichordates and conodonts as well. And Acanthostega and Tulerpeton had precisely four legs underneath, never mind how many digits they sported on the ends. Homoeobox genes may tell us why.

\title{
Variations on an original theme
}

\section{F. Quate}

IN the nine years since its invention, the scanning atomic probe microscope has been adapted in numerous ways so that it can be used to trace not merely surface topography but also surface properties with remarkable resolution. The adaptation, described by Weaver et al. on page 783 of this issue ${ }^{1}$, exploiting the thermoelectric effect to identify individual species, exemplifies the ingenuity now applied in the field.

Early in the 1980s Heini Rohrer and Gerd Binnig discovered that a single atom at the end of a sharp tungsten needle can probe the electronic structure of smooth surfaces. When placed close to the surface and scanned mechanically over it, their atomic probe gave sufficient resolution to define the spatial positions of individual surficial atoms with great precision. But surface scientists need to know more than the location and the chemical nature of the atoms; they also want to know about the environment surrounding the site, the size of the atom's magnetic dipole moment, the size of the electric dipole and much more.

In their new report, Weaver et al. ${ }^{1}$ outline a strategy for acquiring information of this kind, exploiting the thermal voltage generated when a substrate is heated with the energy absorbed from a laser beam. It is well known from optical spectroscopy that such heating is selective: different species absorb energy at dif-

\section{0 years ago \\ REMARKABLE HAILSTONES}

ON p. 43 of the present volume of Nature the following extract is given from a paper by Prof. Houston in the Journal of the Franklin Institute: - "On some of the hailstones, though not on the majority of them, wellmarked crystals of clear transparent ice projected from their outer surfaces for distances ranging from $1 / 8$ to $1 / 4$ of an inch. These crystals, as well as I could observe from the evanescent nature of the material, were hexagonal prisms with clearly cut terminal facets. They resembled the projecting crystals that form so common a lining in geodic masses, in which they have formed by gradual crystallization from the mother-liquor. They differed, however, of course, in being on the outer surface of the spherules."

It is evident from Prof. Houston's paper that this form of hail was unknown to him as it must also have been unknown to many who have propounded theories as to the formation of hail which will not account for it, a service may be rendered to meteorology by the reproduction of the exquisite lithographs of this form of hail given in Prof. Abich's paper, "Ueber krystallinischen Hagel im Thrialethischen gebirge," published at Tiflis in 1871. The hailstones represented all fell on June 9 (21), 1867 , at Bjeloi Kliutsch, a village about twenty ferent wavelengths, so that the result of scanning a heterogeneous surface with a particular wavelength is a variation in the temperature and the chemical potential ${ }^{2}$. As Weaver et al. point out, the tip of a scanning probe and the atoms of the sample are in contact owing to the overlap of their electronic wavefunctions. Electrons flow through the (resistive) contact to equalize the differing chemical potentials and so generate a voltage that is characteristic of the surface atomic species (see figure, over).

The strong interaction between electron waves and optical waves was previously exploited by Gimzewski et $\mathrm{al}^{3}{ }^{3}$, who measured the optical emission from the tunnelling region. Light emission, stimulated by the tunnelling electrons, was first reported by Lambe and McCarthy ${ }^{4}$. It is the result of an inelastic tunnelling process, directly related to the inverse photoelectric effect ${ }^{5}$ in which the electrons in the gap deliver energy to the optical modes. Gimzewski et al. examined a silver film deposited on a silicon substrate and found that the light was associated with surface plasmons (charge density oscillations). The scanning tip perturbs these modes in such a way that they radiate from the vicinity of the tip and the optical emission yields a spatial map useful for characterizing nanometre-sized features.

How can the techniques be extended to

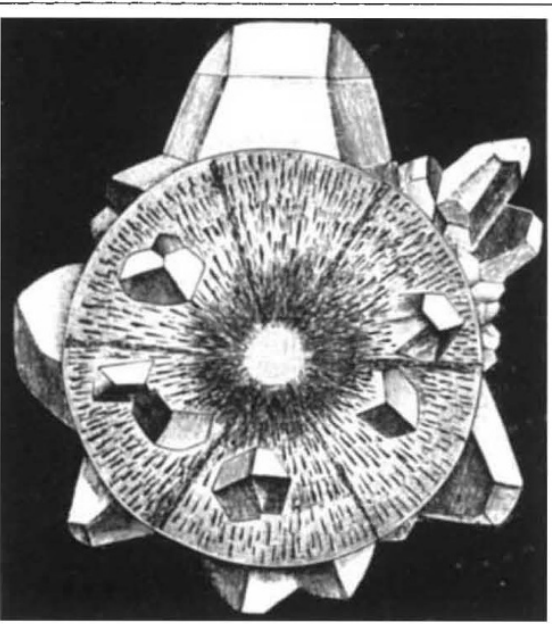

miles south-east of Tiflis, and 12,425 feet above sea-level.

Theories of the formation of hail are almost innumerable. Some - like Prof. Schwedoff's, that hailstones come from interplanetary space - are very droll; but the subject is a very difficult one, and one upon which I do not know of a single good treatise in our language. Possibly, the reproduction of these figures may induce someone to prepare an exhaustive memoir.

G. J. SYMONS

From Nature XLI, 134-135 (1889). 